\title{
Overexpression of Alternatively Spliced Tissue Factor Induces the Pro-Angiogenic Properties of Murine Cardiomyocytic HL-1 Cells
}

\author{
Andreas Eisenreich, PhD; Ulrike Boltzen, PhD; Ronny Malz; \\ Heinz-Peter Schultheiss, MD; Ursula Rauch, MD
}

\begin{abstract}
Background: Tissue factor (TF) is expressed in 2 isoforms: membrane-bound "full length" (fl)TF and soluble alternatively spliced (as)TF. fITF is the major thrombogenic form of TF. Although the function of asTF is poorly understood, it was suggested that asTF contributes to tumor-associated growth and angiogenesis. In the heart of a developing embryo, asTF is expressed much later compared to fITF, but in adult heart, asTF exhibits a distribution pattern similar to that of fITF. Thus, it is possible that asTF may play a role in heart development via proangiogenic signaling. The purpose of the present study was to examine the effects of murine asTF overexpression in murine cardiomyocyte-like HL-1 cells on their pro-angiogenic potential, the chemotaxis of monocytic cells, and the expression of fibroblast growth factor-2 (FGF2), cysteine-rich 61 (Cyr61), and vascular endothelial growth factor (VEGF).

Methods and Results: Expression of FGF2, Cyr61 and VEGF was assessed on reverse transcription-polymerase chain reaction and western blot. Cell migration, proliferation, and endothelial tube formation assays were carried out. It was found that overexpression of murine asTF in $\mathrm{HL}-1$ cells increases their proliferation and proangiogenic properties. The supernatant of murine asTF-overexpressing $\mathrm{HL}-1$ cells induces the chemotaxis of monocytic cells.
\end{abstract}

Conclusions: Overexpression of murine asTF in murine cardiomyocytic cells increases their proliferation, monocyte migration, and pro-angiogenic properties - possibly- mediated by the induction of the pro-migratory and pro-angiogenic factors FGF2, Cyr61 and VEGF. Thus, we propose that murine asTF may serve as a migration- and angiogenesis-promoting factor. (Circ $J$ 2011; 75: 1235-1242)

Key Words: Alternatively spliced tissue factor; Angiogenesis; Chemokines; Growth; Migration

I schemic heart disease is a major cause of mortality. ${ }^{1}$ Myocardial infarction (MI) is a common representation of ischemic heart disease. Reduced systolic compliance, and arrhythmias due to electrical instability of the myocardium are the 2 main consequences of MI. Therefore, improvement of the blood supply to the infarcted myocardium through the formation of microvessels is a potential therapeutic approach to treat MI. ${ }^{2}$ Angiogenesis as well as cell proliferation are crucial for the regenerative formation of new blood vessels. Both processes are potentiated by various bioactive molecules, such as fibroblast growth factor-2 (FGF2), cysteine-rich 61 (Cyr61, CCN1), and vascular endothelial growth factor (VEGF). ${ }^{3-6}$

Tissue factor (TF), a 47-kD transmembrane cell-surface glycoprotein, is primarily known as the initiator of the blood coagulation cascade. ${ }^{7-11}$ TF has been detected in the heart, where it is expressed in various subcompartments, for exam- ple fibroblasts, cardiomyocytes, and cell-derived microparticles. ${ }^{7,9,10,12,13}$ As a result of alternative pre-mRNA splicing, TF is expressed in 2 isoforms: "full length" (fl)TF and alternatively spliced (as)TF. ${ }^{14-19}$ flTF is membrane-bound and comprises the major thrombogenic form of TF. ${ }^{7-9}$ Myocardial flTF protects the heart against spontaneous hemorrhage. ${ }^{20}$ Our group recently showed that flTF as well as asTF are downregulated in the myocardium of patients with dilated cardiomyopathy. ${ }^{21}$ In contrast to flTF, the biologic function of the soluble asTF isoform is not well understood. Although asTF promotes FXa generation in the presence of phospholipids, the pro-coagulant activity of asTF is much lower than that of flTF. $8,14,17,22$ Recently, it was suggested that human asTF may be linked to increased cell proliferation and angiogenesis. ${ }^{23,24}$ Like asTF, flTF was also shown to enforce angiogenesis, chiefly via protease activated receptor (PAR)-

Received August 5, 2010; revised manuscript received December 10, 2010; accepted January 16, 2011; released online March 7,

2011 Time for primary review: 27 days

Charitè-Universitätsmedizin Berlin, Campus Benjamin Franklin, Centrum für Herz- und Kreislaufmedizin, Berlin, Germany

Mailing address: Andreas Eisenreich, PhD, Charitè-Universitätsmedizin Berlin, Campus Benjamin Franklin, Centrum für Herz- und

Kreislaufmedizin, Hindenburgdamm 30, 12200 Berlin, Germany. E-mail: andreas.eisenreich@ charite.de

ISSN-1346-9843 doi:10.1253/circj.CJ-10-0783

All rights are reserved to the Japanese Circulation Society. For permissions, please e-mail: cj@j-circ.or.jp 
2 activation through FVII and FX. ${ }^{25}$

In the course of heart development, when cell proliferation and pro-angiogenic processes are intensified, asTF is expressed at increasingly higher levels compared to relatively constant levels of fITF and, in adult hearts, where developmental processes are complete, asTF and flTF exhibit similar levels and distribution patterns. ${ }^{17}$ These observations suggest that asTF may possibly play a role in developmental and/or regenerative processes, such as angiogenesis and cell proliferation. Here, we show that overexpression of murine asTF in murine cardiomyocytic HL-1 cells induces the expression of pro-angiogenic as well as pro-migratory factors. Moreover, the overexpression of murine asTF is associated with increased cell proliferation, migration of monocytic cells, and endothelial tube formation. These data indicate that murine asTF is a potential pro-migratory and pro-angiogenic factor in the myocardium. We propose that therapeutic induction of asTF in infarcted regions of the heart may enhance regenerative cell proliferation and angiogenesis, thereby improving the outcome of ischemic heart disease.

\section{Methods}

\section{Cell Culture}

Murine cardiomyocyte-like cells (HL-1) and human monocytic cells (THP-1) were cultured in Claycomb medium or RPMI medium (containing 10\% fetal calf serum [FCS], PAA Laboratories, Pasching, Austria), respectively, at $37^{\circ} \mathrm{C}$ in a humidified incubator $\left(5 \% \mathrm{CO}_{2}, 95 \%\right.$ air). Murine microvascular endothelial cells (MHEC5-T) were cultured in DMEM (containing 10\% FCS, PAA Laboratories).

\section{Overexpression Plasmids}

For transfection experiments the plasmid pEGFP-N1 (Becton Dickinson, Heidelberg, Germany) was used. A cDNA construct of murine asTF was cloned into the multiple cloning site of the plasmid. The quality, orientation and identity of the asTF overexpression vector (pEGFP-N1_asTF) were confirmed by automated sequencing. The empty plasmid (pEGFP-N1_LV) was used for control transfections.

\section{Stable Transfection of HL-1 Cells}

A total of $5 \times 10^{6} \mathrm{HL}-1$ cells per well were seeded in 6-well plates. Transfection of pEGFP-N1_asTF or pEGFP-N1_LV was done using Lipofectamin ${ }^{\mathrm{TM}} 2000$ (Invitrogen, Karlsruhe, Germany) following the manufacturer's protocol. Selection of stable transfectants was performed by incubating transfected cells with $800 \mu \mathrm{g} / \mathrm{ml}$ gentamycin (PAA Laboratories). Stable overexpression was continuously verified on quantitative reverse transcription-polymerase chain reaction (RT-PCR).

\section{Real-Time PCR (TaqMan ${ }^{\circledR}$ )}

Real-time PCR using specific gene expression assays for FGF2, Cyr61 and VEGF (Applied Biosystems, Carlsbad, CA, USA) was performed following the manufacturer's instructions.

\section{Semi-Quantitative RT-PCR}

Total RNA was reverse transcribed using AMV (Roche Diagnostics, Mannheim, Germany), and cDNAs encoding FGF2, Cyr61 and VEGF were amplified. Primers used for detection were as follows: FGF2_for, 5'AGCGGCATCACCTCGCTTCC-3'; FGF2_rev, 5'-TGGAAGAAACAGTATGGCCTTCTGTCC-3'; amplicon, 437 bp; Cyr61_for, 5'-GAAGGCAGACCCTGTGAATA-3'; Cyr61_rev, 5'-TG-
GTCTTGCTGCAT TTCTTG-3'; amplicon, 576bp; VEGF_for, 5'-CTGCTCTCTTGGGTCCACTGG-3'; VEGF_rev, 5'-CACCGCCTTGGCTTGTCACAT-3'; amplicons, 562 bp and $634 \mathrm{bp} ; \beta$-actin_for, 5'-TGTACGTAGCCATCCAGGCT-3'; $\beta$-actin_rev, 5'-TTCTCCAGGGAGGAAGAGGA-3'; amplicon, $319 \mathrm{bp}$ (Ocimum Biosolutions, Hyderabad, India). PCR conditions: $95^{\circ} \mathrm{C}, 2 \mathrm{~min}$, and 36 cycles of $95^{\circ} \mathrm{C}, 30 \mathrm{~s} ; 60^{\circ} \mathrm{C}$, $25 \mathrm{~s}$; and $72^{\circ} \mathrm{C}, 1 \mathrm{~min}$.

\section{Western Blotting}

Western blot analysis of HL-1 lysates was performed as previously described. ${ }^{8}$ For protein detection, specific antibodies against FGF2 (Millipore, Schwalbach, Germany), Cyr61 (a kindly gift from Lester F. Lau, University of Illinois, Chicago, IL, USA), VEGF (Abnova, Heidelberg, Germany), phosphorylated as well as total ERK 1/2 and phosphorylated p38 (Cell Signalling Technology, Danvers, MA, USA), total p38 and GAPDH (Calbiochem, Darmstadt, Germany) were used.

\section{In Vitro Cell Migration Assay}

Transwell inserts with $8-\mu \mathrm{m}$ pore size fitted in 24 -well plates (Becton Dickinson) were used. A total of $1 \times 10^{6}$ THP-1 cells were resuspended in $100 \mu \mathrm{l}$ fresh RPMI without FCS. A total of $800 \mu \mathrm{l}$ of the supernatant of asTF-overexpressing HL-1 cells or control HL-1 cells (transfected with empty plasmid) were placed into the lower chamber. FCS-free RPMI medium alone was used as a negative control. Cells were incubated for $48 \mathrm{~h}$ at $37^{\circ} \mathrm{C}$. After that, cells that had migrated into the lower chamber were counted using a Neubauer counting chamber and a Leica DMIL light microscope (Leica, Wetzlar, Germany).

\section{Recombinant Murine TF Proteins}

flTF was obtained from American Diagnostica (Pfungstadt, Germany). Recombinant murine asTF mature peptide was produced in Escherichia coli and re-solubilized from bacterial inclusion bodies (a gift from the laboratory of Dr Vladimir Y. Bogdanov, University of Cincinnati, Cincinnati, OH, USA). For stimulation experiments different concentrations of recombinant (r)asTF or (r)flTF $(0-1,000 \mathrm{nmol} / \mathrm{L})$ were added to the FCS-free medium in the lower chamber to characterize the pro-migratory potential of these factors.

\section{Tube Formation Assay}

Angiogenesis Assay Kit (Millipore, Schwalbach, Germany) was used and experiments were performed following the manufacturer's protocol. In brief, $7.5 \times 10^{3}$ MHEC5-T per well were resuspended in the supernatant of asTF-overexpressing HL-1 cells or cells transfected with empty plasmid. After that, these cells were seeded on $50 \mu \mathrm{l}$ of the ECMatrix ${ }^{\mathrm{TM}}$ in $96-w e l l$ plates and incubated for $7 \mathrm{~h}$ at $37^{\circ} \mathrm{C}$ in humidified air with $5 \% \mathrm{CO}_{2}$. Tube formation by endothelial cells was analyzed using Leica DMIL light microscope (Leica).

\section{Carboxyfluorescein Succinimidyl Ester Cell Proliferation Assays}

HL-1 cells were labeled with carboxyfluorescein succinimidyl ester (CSFE, $5 \mu \mathrm{mol} / \mathrm{L}$ ). After that, cells were washed and resuspended in Claycomb medium (containing 10\% FCS; PAA Laboratories). A total of $1 \times 10^{6} \mathrm{HL}-1$ cells per well were seeded in 12 -well plates and incubated at $37^{\circ} \mathrm{C}$ in humidified air with $5 \% \mathrm{CO}_{2}$. Cell proliferation was measured after $24 \mathrm{~h}$, $48 \mathrm{~h}$ and $72 \mathrm{~h}$ using flow cytometry at $488 \mathrm{~nm} .^{26}$ 


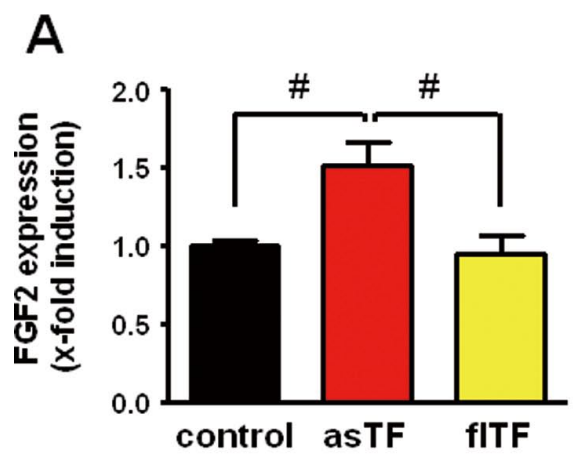

B
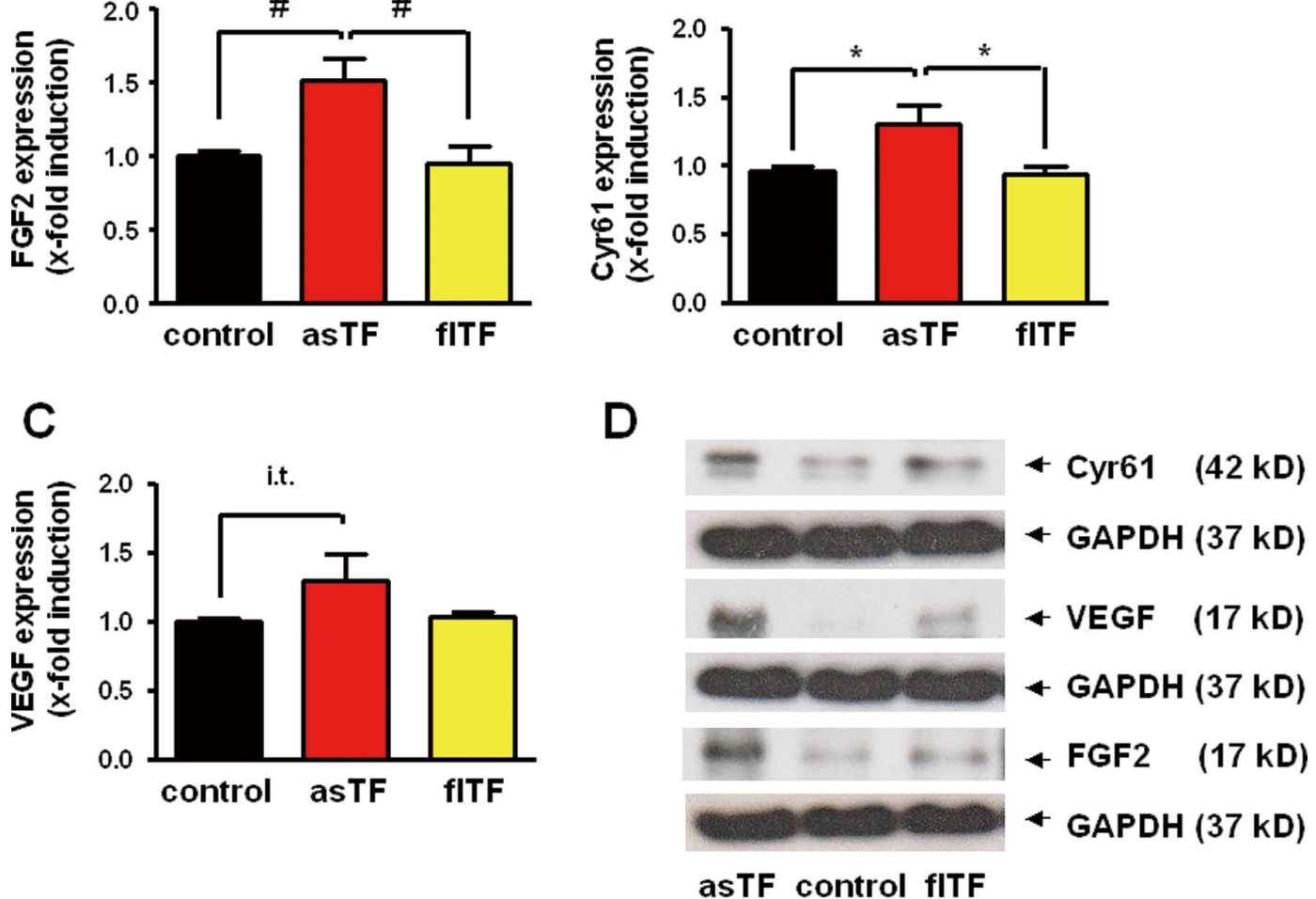

Figure 1. Stable overexpression of alternatively spliced tissue factor (asTF) alters the expression pattern in HL-1 cells. (A-C) Analysis of the mRNA expression of (A) fibroblast growth factor-2 (FGF2), (B) cysteine-rich 61 (Cyr61) and (C) vascular endothelial growth factor (VEGF) in murine cardiomyocyte-like HL-1 cells normalized against GAPDH. Compared are HL-1 cells stably overexpressing asTF or full length TF (fITF), respectively, and control cells transfected with empty plasmid (control). (A-C) Real-time reverse transcription-polymerase chain reaction analyses given as mean \pm SEM of at least 8 independent experiments. i.t., in trend $P<0.15 ;{ }^{*} P<0.05$; and ${ }^{\#} P<0.01$. (D) Western blot analysis of intracellular protein expression of FGF2, Cyr61 and VEGF (representative of at least 3 independent experiments). GAPDH was used as loading control.

\section{Ki67 Cell Proliferation Test}

A total of $1 \times 10^{6} \mathrm{HL}-1$ cells were fixed in paraformaldehyde at $4^{\circ} \mathrm{C}$ overnight. After that, cells were centrifuged and resuspended in phosphate-buffered saline. Cells were permeabilized with $0.1 \%$ TritonX for $5 \mathrm{~min}$, washed and centrifuged. Cells were incubated with an anti-Ki67 antibody for $1 \mathrm{~h}$ at room temperature. Thereafter, cells were washed and incubated with a second anti-mouse-M488 antibody for $45 \mathrm{~min}$ at room temperature. Finally, the cell proliferation was assessed using flow cytometry. ${ }^{26}$

\section{Statistical Analysis}

All data are expressed as mean \pm SEM. Data were analyzed using Student's t-test or 1-way ANOVA. P $\leq 0.05$ was deemed significant.

\section{Results}

Overexpression of the Soluble TF Isoform Induces Expression of Pro-Migratory and Pro-Angiogenic Factors in HL- 1 Cells

Stable overexpression of murine asTF in murine cardiomyocyte-like HL-1 cells induced the expression of the pro-angiogenic and pro-migratory factors at the mRNA and protein levels (Figure 1). Compared to control cells transfected with empty plasmid or cells overexpressing flTF, the mRNA ex- pression of FGF2 (Figure 1A) and Cyr61 (Figure 1B) was significantly increased in asTF-overexpressing HL-1 cells. Moreover, asTF overexpression also induced the mRNA expression of VEGF in these cells compared to control cells and flTF-overexpressing HL-1 cells (Figure 1C). On the protein level, the expression of FGF2, Cyr61, and VEGF was appreciably increased in asTF-overexpressing cardiomyocyte-like HL-1 cells (Figure 1D).

Overexpression of asTF Increases Proliferation of HL-1 Cells Stable overexpression of asTF in HL-1 cells significantly increased cell proliferation, compared to control cells transfected with empty plasmid (Figures 2 A, B). In contrast to stable overexpression, siRNA-mediated downregulation of total TF in HL-1 cells led to a significant reduction of cell proliferation compared to control cells (Figures 2A,B). The inhibitory impact of siRNA on total TF expression was verified on western blotting and semi-quantitative RT-PCR (data not shown). Furthermore, the stimulation of HL-1 cells with (r)asTF induced cell proliferation in a dose-dependent manner (Figure 2C). In contrast to (r)asTF, (r)flTF had no significant impact on cell proliferation (Figure 2D). Treatment of HL-1 cells with lipopolysaccharide (LPS) at equimolar concentrations did not cause the increase in cell proliferation observed with (r)asTF (data not shown). 


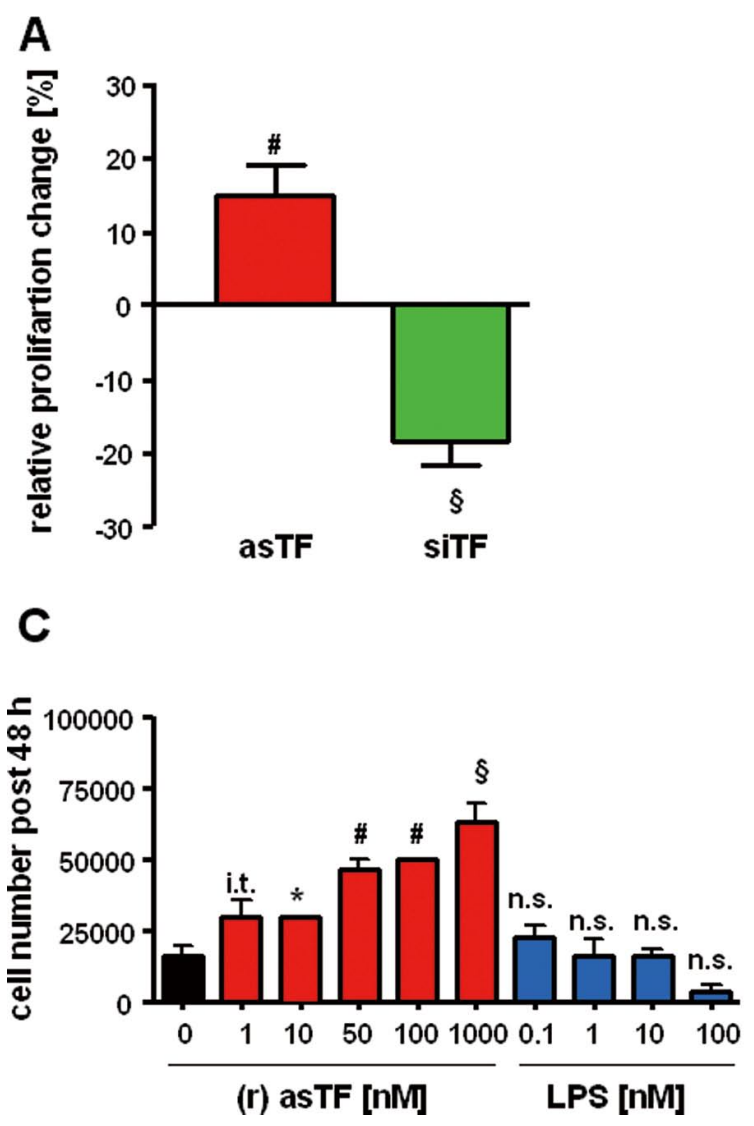

\section{B}
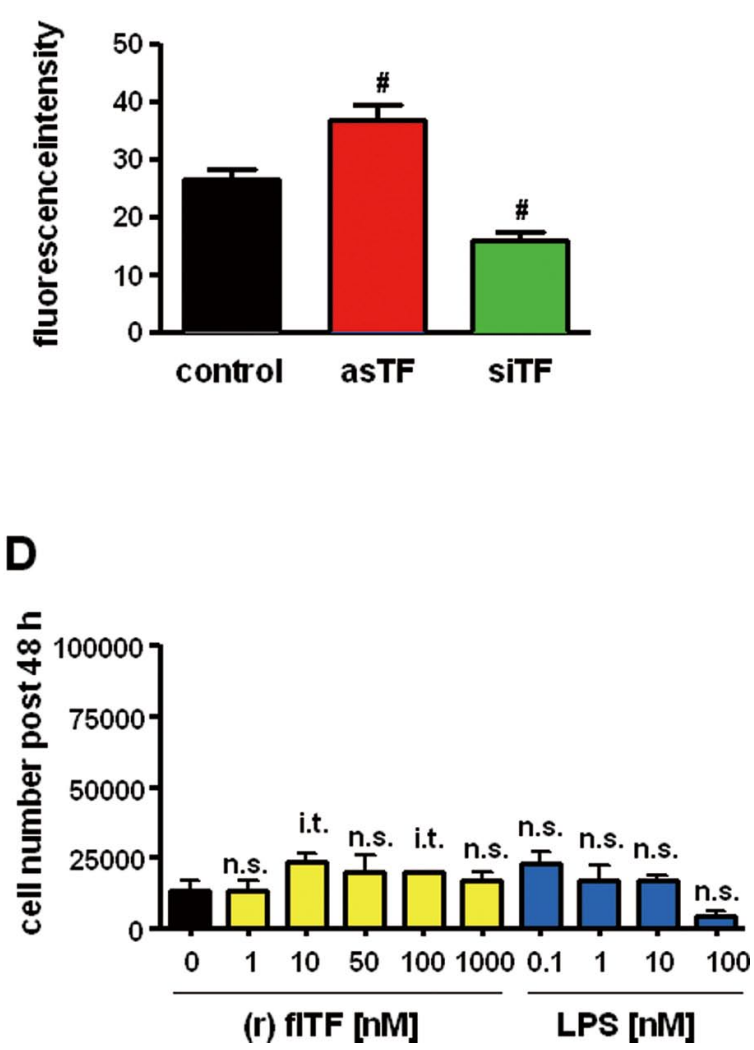

Figure 2. Overexpression of murine alternatively spliced tissue factor (asTF) increases proliferation of murine cardiomyocytic $\mathrm{HL}-1$ cells. Shown is impact of murine asTF overexpression on cell proliferation as $(\mathbf{A})$ the relative proliferation change or (B) increase in fluorescence intensity, respectively. Compared are asTF-overexpressing HL-1 cells (asTF), cells pre-treated with inhibitory siRNAs against total TF (siTF), and HL-1 cells stably transfected with empty plasmid (control). (C, D) Characterization of the influence of $(\mathbf{C})$ recombinant (r)asTF or (D) (r)full length TF [(r)fITF], respectively, on cell proliferation. To test the possible influence of endotoxin contamination, different concentrations of lipopolysaccharide (LPS, 0.1-100 nmol/L) were used. Compared are wild-type $\mathrm{HL}-1$ cardiomyocytic cells treated for $48 \mathrm{~h}$ with different concentrations of ( $r$ )asTF or ( $r$ )fITF ranging from 0 to $1,000 \mathrm{nmol} / \mathrm{L}$. The mean $\pm \mathrm{SEM}$ of $5-8$ independent experiments is shown. i.t., in trend $\mathrm{P}<0.15$; ${ }^{*} \mathrm{P}<0.05$; ${ }^{\#} \mathrm{P}<0.01$; ${ }^{\circledR} \mathrm{P}<0.001$.

Impact of asTF Overexpression on THP-1 Migration

The pro-migratory effect of asTF overexpression on the chemotaxis of monocytic THP-1 cells was analyzed using a Boyden migration chamber. The supernatants of asTF-overexpressing HL-1 cells and control cells were analyzed with regard to the pro-migratory potential. The supernatant of asTF-overexpressing HL-1 cells significantly increased the migration of THP-1 cells, compared to the supernatant of HL-1 cells transfected with empty plasmid (Figure $3 \mathbf{A}$ ). Treatment with (r)asTF induced the migration of THP-1 cells in a dosedependent manner (Figure 3B). A significant induction of chemotaxis was achieved when stimulating the cells with $1 \mathrm{nmol} / \mathrm{L}$ up to $1,000 \mathrm{nmol} / \mathrm{L}$ (r)asTF. In contrast to (r)asTF, (r)flTF had no significant influence on THP-1 migration (Figure 3C). Treatment of cells with LPS at equimolar concentrations did not cause the increase in THP-1 migration observed with (r)asTF (Figures 3B, C).

\section{Supernatant of asTF-Overexpressing HL-1 Cells Increases Endothelial Tube Formation}

To evaluate the possible pro-angiogenic effect of asTF overexpression in HL-1 cells, the impact of the supernatant of these cells on tube formation by murine endothelial cells was assessed (Figure 4). The supernatant of stable transfected asTF-overexpressing HL-1 cells significantly increased the tube formation activity of murine endothelial MHEC5-T cells compared to the supernatant of control cells, indicating that asTF may potentiate pro-angiogenic potential of HL-1 cells (Figure 4).

\section{Possible Regulatory Pathways Involved in the asTF-Induced Effects on the Expression of Chemotaxis- and Angiogenesis- Promoting Factors in HL-1 Cells}

In order to delineate the regulatory pathways underlying the observed asTF-associated effects on HL-1 cells, the phosphorylation states of the signaling molecules involved in the regulation of VEGF, FGF2, and Cyr61 expression were examined. Stimulation of HL-1 cells with $100 \mathrm{nmol} / \mathrm{L}$ (r)asTF reduced the phosphorylation of ERK $1 / 2$ but increased the phosphorylation of p38 as compared to non-treated control cells (Figure 5A). In cells overexpressing as TF the phosphorylation pattern of ERK 1/2 and p38 was modified in the same manner as seen after stimulation with (r)asTF. No impact of (r)asTF or asTF overexpression in HL-1 cells on the phos- 

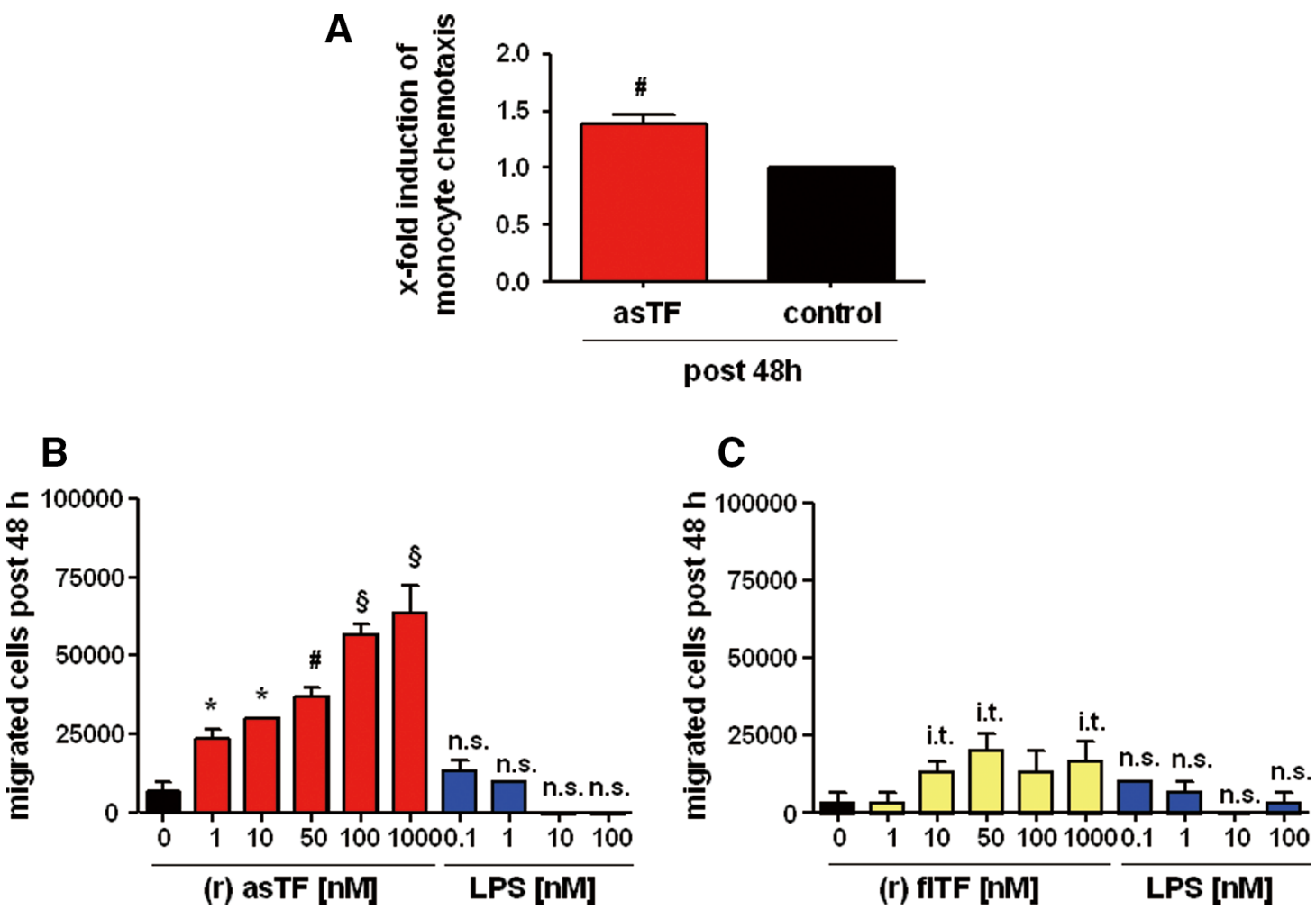

Figure 3. The supernatant of alternatively spliced tissue factor (asTF)-overexpressing HL-1 cells increases the migration of monocytic THP-1 cells. (A) Fold induction of chemotaxis of THP-1 cells treated with the supernatant of asTF-overexpressing cardiomyocytic cells (asTF), or HL-1 cells stably transfected with empty plasmid (control), respectively. (B,C) Impact of different concentrations of $(\mathbf{B})$ recombinant ( $r$ )asTF or $(\mathbf{C})$ ( $r$ )full length TF $[(r) f I T F]$ ranging from 0 to $1,000 \mathrm{nmol} / \mathrm{L}$ on the number of migrated THP-1 cells after $48 \mathrm{~h}$. To test the impact of endotoxin contamination, different concentrations of lipopolysaccharide (LPS, $0.1-100 \mathrm{nmol} / \mathrm{L}$ ) were used. Mean \pm SEM of at least 3 independent experiments. i.t., in trend $P<0.15$; ${ }^{*} P<0.05$; ${ }^{\#} P<0.01$; $\S \mathrm{P}<0.001$.

phorylation state of JNK was detectable (data not shown). Moreover, the inhibition of ERK $1 / 2$ and p38 differentially modified the mRNA expression pattern of VEGF, FGF2 and Cyr61 in non-treated wild-type HL-1 cells, as well as wild-type HL-1 cells stimulated with $100 \mathrm{nmol} / \mathrm{L}$ (r)asTF (Figure 5B).

\section{Discussion}

In the present study we report for the first time that overexpression of asTF in the murine cardiomyocyte-like cell line HL-1 increases the expression of pro-migratory as well as proangiogenic factors FGF2, Cyr61 and VEGF. Furthermore, we show that asTF overexpression increases cell proliferation and pro-angiogenic properties of HL-1 cells.

\section{asTF Overexpression Increases Proliferation of HL-1 Cells}

In the present study we found the overexpression of murine asTF to be associated with increased proliferation of murine cardiomyocyte-like HL-1 cells. These results are in line with the data of Hobbs et al, who reported that human asTF overexpression in pancreatic cancer cells increases tumor cell proliferation. ${ }^{23}$ Moreover, overexpression of murine asTF in HL-1 cells was associated with an increased expression of FGF2, Cyr61 and VEGF. All these factors are known to increase cell proliferation. ${ }^{3-6}$

We therefore hypothesize that the increase in cell prolif-

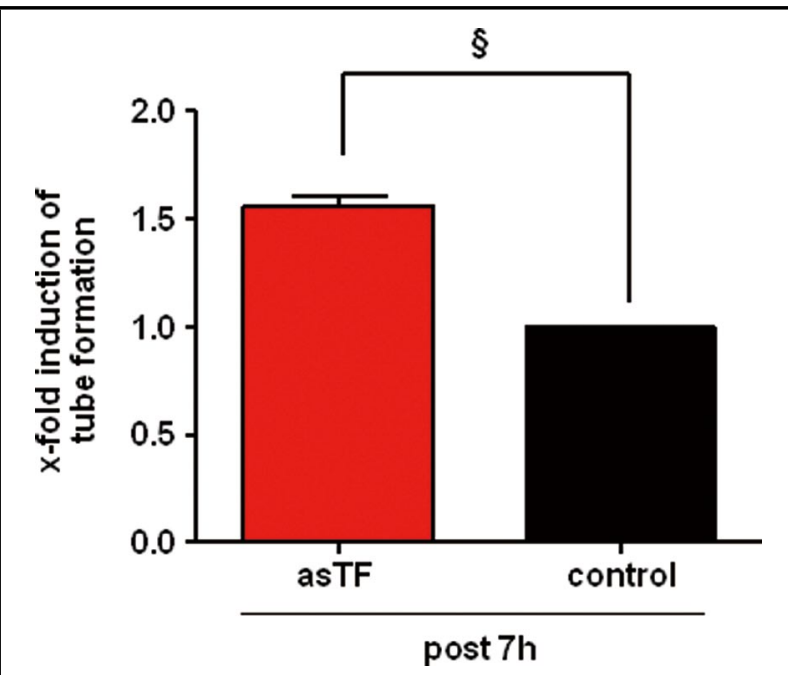

Figure 4. Impact of the supernatant from alternatively spliced tissue factor (asTF)-overexpressing HL-1 cells on endothelial tube formation. Fold induction of tube formation of MHEC5-T cells treated with the supernatant of asTF-overexpressing HL-1 cells (asTF), or cells stably transfected with empty control plasmid (control), respectively. Mean \pm SEM of at least 3 independent experiments. ${ } \mathrm{P}<0.001$. 
A
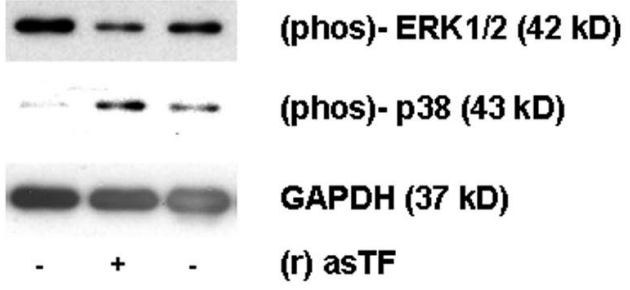

wt asTF

B

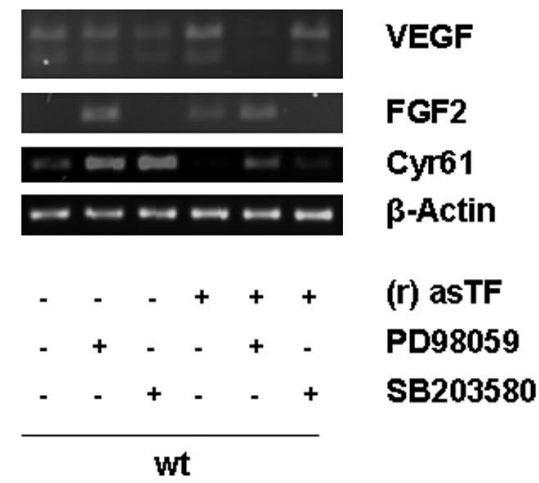

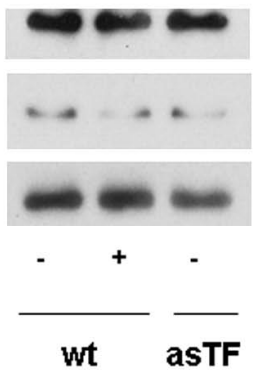

(total)- ERK1/2 (42 kD)

(total)- p38 (43 kD)

GAPDH (37 kD)

(r) asTF

C

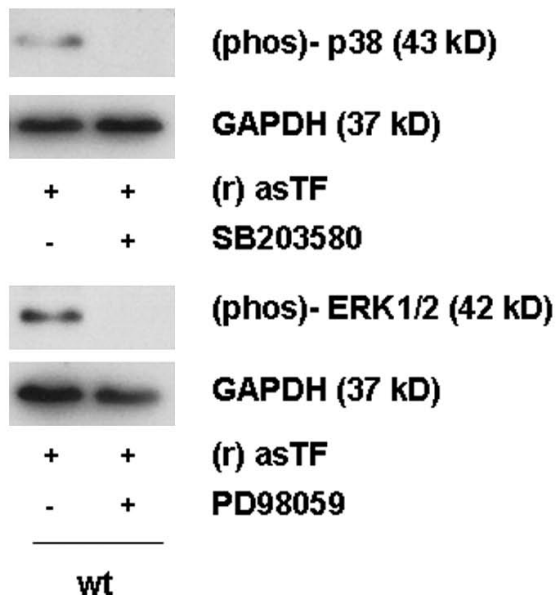

Figure 5. Effect of murine alternatively spliced tissue factor (asTF) on regulatory signaling pathways in $\mathrm{HL}-1 \mathrm{cells}$. (A) Impact of recombinant ( $r$ )asTF or stable overexpression of asTF in HL-1 cells, respectively, on (Left) the phosphorylation state of ERK $1 / 2$ and p38 and on (Right) total ERK $1 / 2$ and total p38, respectively. Stimulation was carried out with $100 \mathrm{nmol} / \mathrm{L}$ ( $\mathrm{r}$ )asTF, and compared to asTF-overexpressing HL-1 cells (asTF). Non-treated wild-type cells were used as controls (control). (B) Impact of pharmacologic inhibition of ERK $1 / 2$ with $10 \mu \mathrm{mol} / \mathrm{L}$ PD98059 and of p38 with $10 \mu \mathrm{mol} / \mathrm{L}$ SB203580 on the mRNA expression of vascular endothelial growth factor (VEGF), fibroblast growth factor-2 (FGF2) and cysteine-rich 61 (Cyr61) in wild-type (wt) HL-1 cells, $2 \mathrm{~h}$ after stimulation of cells with $100 \mathrm{nmol} / \mathrm{L}$ (r)asTF. (C) Representative western blot analysis of the influence of pharmacologic inhibitors PD98059 (10 $\mu \mathrm{mol} / \mathrm{L})$ or SB203580 (10 $\mu \mathrm{mol} / \mathrm{L})$, respectively, on the phosphorylation of ERK 1/2 and p38.

eration associated with the overexpression of asTF in cardiomyocytic HL-1 cells is, at least in part, mediated by an asTF-induced enhancement of the expression of the proliferation-promoting factors FGF2, Cyr61 and VEGF. This hypothesis was substantiated by results of western blot analyses and RT-PCR. ERK 1/2 as well as p38 are known to be involved in the regulation of expression of FGF2, Cyr61 and VEGF. ${ }^{27}$ These signaling factors are, among others, downstream effectors of PAR-1 and PAR-2. ${ }^{27,28}$ Our data showed that the stimulation of HL-1 cells with (r)asTF as well as the stable asTF overexpression in these cells, modified the phosphorylation state of ERK 1/2 and p38. This is in line with the results of van den Berg et al showing (r)asTF to induce the phosphorylation of p38 in human endothelial cells. ${ }^{24}$ Blocking of ERK 1/2 as well as p38 altered the expression pattern of FGF2, Cyr61 and VEGF in non-stimulated and (r)asTFstimulated HL-1 cells. Thus, the asTF-induced impact on the expression of the pro-migratory factors FGF2, Cyr61 and VEGF seems to be mediated via ERK $1 / 2$ and p38 phosphorylation, although it cannot be excluded that additional signaling cascades may also play a role.

Membrane-bound flTF complexes and activates FVII/
FVIIa, thereby initiating the blood coagulation cascade. ${ }^{29-31}$ The fITF:FVII complex was demonstrated to induce the expression of Cyr61 as well as VEGF. ${ }^{32,33}$ Moreover, this complex was also suggested to increase the expression of FGF2 indirectly via activation of PAR signaling. ${ }^{34}$ Bogdanov et al showed that the sequence involved in FVIIa binding is maintained in human and murine asTF. ${ }^{14,17}$ It is therefore probable that asTF may be able to bind and activate FVII/FVIIa, leading to associated downstream signaling events. ${ }^{14,30,35}$ In our experiments we used medium containing FCS. Neither data from the manufacturer nor western blot analyses showed FVII to be present in the culture medium or the FCS. But we cannot exclude the possibility that FVII was involved in the asTF-induced effects on HL-1 cells. Therefore, we analyzed whether recombinant (r)asTF or (r)flTF induce HL-1 cell proliferation in medium without FCS. The results clearly show that (r)asTF but not (r)flTF induces the cell proliferation of cardiomyocytic cells in a dose-dependent manner. Van den Berg et al found intratumoral human asTF protein concentrations to be in the range of $25-75 \mathrm{nmol} / \mathrm{L} .{ }^{24}$ Moreover, they showed highly pure and endotoxin-free human recombinant asTF to induce cell migration of human endothelial cells 
already at a concentration of $1 \mathrm{nmol} / \mathrm{L}$. In line with these observations, we found murine (r)asTF to stimulate cell proliferation of cardiomyocyte-like HL- 1 cells at $1 \mathrm{nmol} / \mathrm{L}$, which is likely to comprise a pharmacologically relevant concentration. Thus, asTF may directly or indirectly induce the expression of FGF2, Cyr61 and VEGF in murine asTF-overexpressing HL-1 cells, thereby contributing to increased cell proliferation.

\section{Overexpression of asTF in HL-1 Cells Induces Migration of Monocytic Cells}

Herein it was shown that the supernatant of murine asTFoverexpressing cardiomyocyte-like HL-1 cells promotes the chemotaxis of THP-1 cells. In line with our data, van den Berg et al found that human asTF induces migration of human endothelial cells via integrin signaling. ${ }^{24}$ In this study, no direct interaction of asTF with integrins could be demonstrated. Therefore, it is possible that the migration-promoting effect of the soluble murine TF isoform was not directly mediated via asTF-integrin interactions. Further studies are needed to investigate whether murine asTF ligates integrins, similar to human as TF. ${ }^{24}$

The supernatant of asTF-overexpressing HL-1 cells increases the chemotaxis of THP-1 cells, and asTF overexpression is associated with an increased expression of the secreted and soluble pro-migratory factors FGF2, Cyr61 and VEGF. The pro-migratory effect of FGF2, Cyr61, as well as VEGF, was previously demonstrated. ${ }^{3-6,32,36}$ As aforementioned, we tested whether different concentrations of (r)asTF or (r)flTF also induce THP-1 chemotaxis. We found (r)asTF but not (r)flTF to induce cell migration in a dose-dependent manner, even at low, more physiologic, concentrations $(1 \mathrm{nmol} / \mathrm{L})$. These observations are in line with the data of van den Berg et al, which demonstrated (r)asTF to induce the migration of human endothelial cells at $1 \mathrm{nmol} / \mathrm{L} .{ }^{24}$ Moreover, from the results of our migration experiments we can estimate that the intensity of chemoattraction achieved by stable asTF overexpression is comparable with the induction of cell migration achieved with $10-50 \mathrm{nmol} / \mathrm{L}$ (r)asTF. There is little information available regarding the physiologic or pathophysiologic levels of asTF in human or murine organisms. Van den Berg et al showed $25-75 \mathrm{nmol} / \mathrm{L}$ to be a pathophysiologically relevant intratumoral concentration range of asTF in patients suffering from cervical cancer. ${ }^{24}$ Therefore, the concentration reached by asTF overexpression in the present experiments is likely to be within this, pathophysiologically relevant, range. In sum, our data suggest that murine asTF is pro-migratory, and that this property of asTF is possibly mediated by increasing the expression of the pro-migratory factors FGF2, Cyr61 and VEGF.

\section{asTF Overexpression in HL-1 Cells Potentiates Endothelial Tube Formation}

Recently, van den Berg et al showed that human asTF induces tube formation by human endothelial cells in vitro as well as sprouting in vivo. ${ }^{24}$ Here, the supernatant of murine asTF-overexpressing cardiomyocytic HL-1 cells was found to induce the tube formation by murine microvascular endothelial cells. These observations are in full agreement with the results of van den Berg et al. ${ }^{24}$

As mentioned earlier, asTF overexpression in HL-1 cells was associated with an increased expression of FGF2, Cyr61 as well as VEGF. All these factors are known to be very proangiogenic. ${ }^{3-6,37,38}$ Therefore, it is probable that the stimulation of endothelial tube formation by the supernatant of
asTF-overexpressing HL-1 cells is due to the increased expression of FGF2, Cyr61 and VEGF.

In summary, the present results indicate that the induction of cell proliferation, monocyte chemotaxis, and endothelial tube formation - events elicited by the overexpression of murine asTF in cardiomyocyte-like HL-1 cells - are likely mediated by an increased expression of FGF2, Cyr61 and VEGF. These processes appear to be mediated by signaling cascades involving ERK 1/2 and p38. Thus, we propose that asTF is a potential chemotaxis- and angiogenesis-promoting factor that participates in developmental and regenerative processes, such as angiogenesis.

\section{Acknowledgments}

This work was supported by grants from the Deutsche Forschungsgemeinschaft (DFG) (SFB-TR19 to U.R., W.P. and H.-P.S.). The authors thank Dr Vladimir Y. Bogdanov and Richard Godby (University of Cincinnati) for providing a sample of recombinant murine asTF, and for their help in the revision of the manuscript.

\section{References}

1. Takahashi M. Role of the SDF-1/CXCR4 system in myocardial infarction. Circ J 2010; 74: 418-423.

2. Takeda Y, Uemura S, Iwama H, Imagawa K, Nishida T, Onoue K, et al. Treatment with recombinant placental growth factor (PlGF) enhances both angiogenesis and arteriogenesis and improves survival after myocardial infarction. Circ J 2009; 73: 1674-1682.

3. Katsumoto M, Shingu T, Kuwashima R, Nakata A, Nomura S, Chayama K. Biphasic effect of HMG-CoA reductase inhibitor, pitavastatin, on vascular endothelial cells and angiogenesis. Circ $J$ 2005; 69: $1547-1555$.

4. Yu P, Passam FH, Yu DM, Denyer G, Krilis SA. Beta2-glycoprotein I inhibits vascular endothelial growth factor and basic fibroblast growth factor induced angiogenesis through its amino terminal domain. J Thromb Haemost 2008; 6: 1215-1223.

5. Grote K, Salguero G, Ballmaier M, Dangers M, Drexler H, Schieffer $\mathrm{B}$. The angiogenic factor CCN1 promotes adhesion and migration of circulating CD34+ progenitor cells: Potential role in angiogenesis and endothelial regeneration. Blood 2007; 110: 877-885.

6. Sakamoto H, Sakamaki T, Kanda T, Tsuchiya Y, Sato M, Sato H, et al. Vascular endothelial growth factor is an autocrine growth factor for cardiac myxoma cells. Circ J 2004; 68: 488-493.

7. Giesen PL, Rauch U, Bohrman B, Kling D, Roque M, Fallon JT, et al. Blood-borne tissue factor: Another view of thrombosis. Proc Natl Acad Sci USA 1999; 96: 2311-2315.

8. Szotowski B, Antoniak S, Poller W, Schultheiss HP, Rauch U. Procoagulant soluble tissue factor is released from endothelial cells in response to inflammatory cytokines. Circ Res 2005; 96: 1233-1239.

9. Rauch U, Bonderman D, Bohrmann B, Badimon JJ, Himber J, Riederer MA, et al. Transfer of tissue factor from leukocytes to platelets is mediated by CD15 and tissue factor. Blood 2000; 96: $170-175$.

10. Rauch U, Nemerson Y. Tissue factor, the blood, and the arterial wall. Trends Cardiovasc Med 2000; 10: 139-143.

11. Mackman N. The many faces of tissue factor. $J$ Thromb Haemost 2009; 7(Suppl 1): 136-139.

12. Antoniak S, Boltzen U, Eisenreich A, Stellbaum C, Poller W, Schultheiss HP, et al. Regulation of cardiomyocyte full-length tissue factor expression and microparticle release under inflammatory conditions in vitro. J Thromb Haemost 2009; 7: 871-878.

13. Eisenreich A, Celebi Ö, Goldin-Lang P, Schultheiss HP, Rauch U. Upregulation of tissue factor expression and thrombogenic activity in human aortic smooth muscle cells by irradiation, rapamycin and paclitaxel. Int Immunopharmacol 2008; 8: 307-311.

14. Bogdanov VY, Balasubramanian V, Hathcock J, Vele O, Lieb M, Nemerson Y. Alternatively spliced human tissue factor: A circulating, soluble, thrombogenic protein. Nat Med 2003; 9: 458-462.

15. Eisenreich A, Bogdanov VY, Zakrzewicz A, Pries A, Antoniak S, Poller W, et al. Cdc2-like kinases and DNA topoisomerase I regulate alternative splicing of tissue factor in human endothelial cells. Circ Res 2009; 104: 589-599.

16. Tardos JG, Eisenreich A, Deikus G, Bechhofer DH, Chandradas S, Zafar U, et al. SR proteins ASF/SF2 and SRp55 participate in tissue factor biosynthesis in human monocytic cells. J Thromb Haemost 2008; 6: $877-884$. 
17. Bogdanov VY, Kirk RI, Miller C, Hathcock JJ, Vele S, Gazdoiu $\mathrm{M}$, et al. Identification and characterization of murine alternatively spliced tissue factor. J Thromb Haemost 2006; 4: 158-167.

18. Eisenreich A, Malz R, Pepke W, Ayral Y, Poller W, Schultheiss $\mathrm{HP}$, et al. Role of the phosphatidylinositol 3-kinase/protein kinase $B$ pathway in regulating alternative splicing of tissue factor mRNA in human endothelial cells. Circ J 2009; 73: 1746-1752.

19. Eisenreich A, Rauch U. PI3K inhibitors in cardiovascular disease. Cardiovasc Ther 2011; 29: 29-36.

20. Pawlinski R, Tencati M, Holscher T, Pedersen B, Voet T, Tilley RE, et al. Role of cardiac myocyte tissue factor in heart hemostasis. J Thromb Haemost 2007; 5: 1693-1700.

21. Szotowski B, Goldin-Lang P, Antoniak S, Bogdanov VY, Pathirana $\mathrm{D}$, Pauschinger $\mathrm{M}$, et al. Alterations in myocardial tissue factor expression and cellular localization in dilated cardiomyopathy. $J A m$ Coll Cardiol 2005; 45: 1081 - 1089.

22. Yu JL, Rak JW. Shedding of tissue factor (TF)-containing microparticles rather than alternatively spliced TF is the main source of TF activity released from human cancer cells. J Thromb Haemost 2004; 2: 2065-2067.

23. Hobbs JE, Zakarija A, Cundiff DL, Doll JA, Hymen E, Cornwell $\mathrm{M}$, et al. Alternatively spliced human tissue factor promotes tumor growth and angiogenesis in a pancreatic cancer tumor model. Thromb Res 2007; 120(Suppl 2): S13-S21.

24. van den Berg YW, van den Hengel LG, Myers HR, Ayachi O, Jordanova E, Ruf W, et al. Alternatively spliced tissue factor induces angiogenesis through integrin ligation. Proc Natl Acad Sci USA 2009; 106: 19497-19502.

25. Kasthuri RS, Taubman MB, Mackman N. Role of tissue factor in cancer. J Clin Oncol 2009; 27: 4834-4838

26. Eisenreich A, Boltzen U, Poller W, Schultheiss HP, Rauch U. Effects of the Cdc2-like kinase-family and DNA topoisomerase I on the alternative splicing of eNOS in TNF-alpha-stimulated human endothelial cells. Biol Chem 2008; 389: $1333-1338$.

27. Bluff JE, Brown NJ, Reed MW, Staton CA. Tissue factor, angiogenesis and tumour progression. Breast Cancer Res 2008; 10: 204.

28. Ahamed J, Versteeg HH, Kerver M, Chen VM, Mueller BM, Hogg $\mathrm{PJ}$, et al. Disulfide isomerization switches tissue factor from coagulation to cell signaling. Proc Natl Acad Sci USA 2006; 103: 13932
13937.

29. Versteeg $\mathrm{HH}$, Ruf W. Tissue factor coagulant function is enhanced by protein-disulfide isomerase independent of oxidoreductase activity. J Biol Chem 2007; 282: 25416-25424.

30. Szotowski B, Antoniak S, Rauch U. Alternatively spliced tissue factor: A previously unknown piece in the puzzle of hemostasis. Trends Cardiovasc Med 2006; 16: 177-182.

31. Szotowski B, Antoniak S, Goldin-Lang P, Tran QV, Pels K, Rosenthal P, et al. Antioxidative treatment inhibits the release of thrombogenic tissue factor from irradiation- and cytokine-induced endothelial cells. Cardiovasc Res 2007; 73: 806-812.

32. Pendurthi UR, Allen KE, Ezban M, Rao LV. Factor VIIa and thrombin induce the expression of Cyr61 and connective tissue growth factor, extracellular matrix signaling proteins that could act as possible downstream mediators in factor VIIa $\mathrm{x}$ tissue factorinduced signal transduction. J Biol Chem 2000; 275: 14632-14641.

33. Ollivier V, Bentolila S, Chabbat J, Hakim J, de Prost D. Tissue factor-dependent vascular endothelial growth factor production by human fibroblasts in response to activated factor VII. Blood 1998; 91: $2698-2703$.

34. Chu AJ. Tissue factor mediates inflammation. Arch Biochem Biophys 2005; 440: $123-132$.

35. Ahamed J, Versteeg HH, Kerver M, Chen VM, Mueller BM, Hogg $\mathrm{PJ}$, et al. Disulfide isomerization switches tissue factor from coagulation to cell signaling. Proc Natl Acad Sci USA 2006; 103: $13932-13937$

36. Takeshita Y, Katsuki Y, Katsuda Y, Kai H, Saito Y, Arima K, et al. Vitamin $\mathrm{C}$ reversed malfunction of peripheral blood-derived mononuclear cells in smokers through antioxidant properties. Circ J 2008; 72: $654-659$.

37. Huang Y, Marui A, Sakaguchi H, Esaki J, Arai Y, Hirose K, et al. Sustained release of prostaglandin E1 potentiates the impaired therapeutic angiogenesis by basic fibroblast growth factor in diabetic murine hindlimb ischemia. Circ J 2008; 72: 1693-1699.

38. Chen N, Leu SJ, Todorovic V, Lam SC, Lau LF. Identification of a novel integrin alphavbeta3 binding site in CCN1 (CYR61) critical for pro-angiogenic activities in vascular endothelial cells. $J$ Biol Chem 2004; 279: 44166-44176. 